\title{
Lipid Droplet Biogenesis: a mystery "unmixing"?
}

Mike F. Renne*, Yoel A. Klug*, Pedro Carvalho

Sir William Dunn School of Pathology, University of Oxford, South Parks Road, Oxford OX1 3RE, UK

*These authors equally contributed to this manuscript

Correspondence should be sent to:

Mike F. Renne (mike.renne@path.ox.ac.uk),

Yoel A. Klug (yoel.klug@path.ox.ac.uk) and

Pedro Carvalho (pedro.carvalho@path.ox.ac.uk)

ORCID identifier:

M.F.R. 0000-0003-0508-2298

Y.A.K. 0000-0001-9775-3161

P.C. 0000-0002-9691-5277 


\begin{abstract}
Lipid droplets (LDs) are versatile organelles with central roles in lipid and energy metabolism in all eukaryotes. They primarily buffer excess fatty acids by storing them as neutral lipids, mainly triglycerides and steryl esters. The neutral lipids form a core, surrounded by a unique phospholipid monolayer coated with a defined set of proteins. Thus, the architecture of LDs sets them apart from all other membrane-bound organelles. The origin of LDs remained controversial for a long time. However, it has become clear that their biogenesis occurs at the endoplasmic reticulum (ER) and is a lipid driven process. LD formation is intiatied by the demixing of neutral lipids from membrane phospholipids, leading to the formation of a neutral lipid "lens" like structure between the leaflets of the ER bilayer. As this lens grows and it buds out of the membrane towards the cytosol to give rise to a LD. Recent biophysical and cell biological experiments indicate that LD biogenesis occurs at specific ER domains. These domains are enriched in various proteins required for normal LD formation and possibly have a lipid composition distinct from the remaining ER membrane. Here, we describe the prevailing model for LD formation and discuss recent insights on how proteins organize ER domains involved in LD biogenesis.
\end{abstract}

\title{
Keywords
}

Lipid Droplet, Endoplasmic Reticulum, Seipin, FIT2, Pex30, Ldo45, LDAF1, Promethin, Neutral Lipids, Lipid Metabolism 


\section{Introduction}

Lipid droplets (LDs) are found in virtually every eukaryotic cell and are the prime organelle for storage of fatty acids in the form of neutral lipids (i.e. fat or oil). The two major neutral lipid classes stored in LDs are, triglycerides (triacylglycerol, TAG) and steryl esters (SE), which consist of fatty acyl chains esterified to glycerol or sterol respectively. In LDs, these hydrophobic TAG and SE molecules define the neutral lipid core, which is surrounded by a lipid monolayer [1,2], coated with a variety of LD-specific proteins. Thus, in contrast to all other membrane-bound organelles, LDs consist of a unique monolayer membrane and have no aqueous lumen.

LDs are highly dynamic [3-6]. As storage organelles, LD number and size is influenced by the nutrient availability. Under nutrient-rich conditions, such as abundance of fatty acids, LDs increase and expand. On the contrary, during periods of scarcity, the fatty acids stored in LDs can be utilized as building blocks for membrane synthesis or for energy production (by fatty acid $\beta$-oxidation). LD formation can also be triggered independently of exogenous nutrients. For example, when cells are confronted with a sudden rise of cellular free fatty acids, e.g. upon pronounced autophagy, LDs expand. Furthermore, perturbations to membrane trafficking or generic ER stress can also induce LD formation and expansion. These observations suggest that LDs have a general capacity to buffer fatty acid levels and protect the endomembrane system, thus averting lipotoxicity. Recently, changes in cellular metabolism were shown shown to affect LD distribution within the cell and their interaction with other organelles [7-9]. While most LDs localize to the cytoplasm, LDs also appear in the nucleus. However, the function of nuclear LDs is still enigmatic $[10,11]$.

Despite their similar architecture, LD size and number can vary dramatically within cell types. Saccharomyces cerevisiae have typically 7-12 LDs per cell with $200-500 \mathrm{~nm}$ in diameter, whereas the oleaginous yeast Yarrowia lypolitca LDs are rougly 2 fold bigger. In mammalian cells the variability in LD size is even higher. In non-adipocyte cells, LDs have 1-4 $\mu \mathrm{m}$ in diameter, whereas in brown adipocytes LDs can reach up to $10 \mu \mathrm{m}$. Most strikingly, white adipocytes, specialized in triglyceride storage, have a single, very large LD with a diameter of over $100 \mu \mathrm{m}$. Often, these differences in the size correlate with distinct LD protein composition. LD heterogeneity is also seen within individual cells but the significance of these observations is still not fully understood. A recent study showed that two populations of LDs co-exist in cells from the Drosophila melanogaster fat body. While large LDs distributed centrally, a population of smaller LDs was observed located more peripherally, close to the plasma membrane [12]. Interestigly, these morphologically distinct populations appear to serve different metabolic purposes within these cells.

In addition to neutral lipid storage, LDs have been implicated in a wide range of other processes. For example, in Drosophila embryos, the LD surface serve as a depot for histones that appear to have two distinct functions; LD-mobilized histones assemble into chromatin in rapidly dividing nuclei of the embryo [13] and are a source of antimicrobial peptides [14]. Certain proteins marked for degradation by the proteasome also appear to localize to LDs, however the role of LDs in proteostais remains enigmatic [15-18]. LDs can also serve as a storage site for hydrophobic small molecules. For example, certain hydrophobic antibiotics accumulate in LDs and modulate cellular susceptibility to bacterial infection $[19,20]$. Finally, LDs are also important in the life cycle of intracellular 
pathogens, including viruses and parasitic eukaryotes [21]. In these cases, LDs provide a cellular platform for viral replication, as in the case of the hepatitis $C$ virus [22], or are utilized to fuel the demanding energy requirements of virus replication [23,24]. Although not exhaustive, this list illustrates the versatility of LDs and highlights the central role of this organelle in cell and organismal physiology (for further reading on these topics, we refer to recent reviews $[3,25]$ and references therein). Due to the plethora of cellular functions of LDs, it is not surprising that LD defects are associated with a variety of human pathologies [26].

In recent years, there have been major advances in understanding the functions and dynamics of LDs. Although the molecular mechanisms by which these organelles form remain enigmatic, an outline of the LD biogenesis appears consensual. It is well established that LDs form at the surface of the ER and bud towards the cytosolic face of the ER membrane. Studies using in silico and in vitro biophysical approaches revealed important features of LD biogenesis and highlighted the relevance of specific lipids for the process. Several proteins required for correct LD biogenesis have also been identified through biochemical and genetic approaches. Perhaps not surprisingly, some of the identified proteins appear to bind and/or modify lipids. Here, we review these recent findings. In particular, we explore how proteins and lipids can locally alter membrane properties to facilitate LD biogenesis.

\section{A model of LD biogenesis}

The formation of LDs in the ER is initiated with the synthesis of neutral lipids, mainly TAG and SE. TAG and SEs are formed by acylation of their respective precursors; diacylglycerol (DAG) and sterols (mainly ergosterol and zymosterol in yeast [27], and cholesterol in mammals [28]). Consistently, yeast cells lacking the enzymes involved in the synthesis of TAG and SE are devoid of LDs [29,30]. On the other hand, expression of any individual neutral lipid biosynthetic enzyme is sufficient for LD formation $[27,29]$, indicating that the presence of either TAG or SE can drive LD formation. Recently it was shown that synthesis of retinol-esters, another class of neutral lipids, can also drive LD formation [31]. In neutral-lipid deficient yeast, expression of an exogenous retinol-ester acyl transferase triggered LD formation upon supplementation of its substrate retinol. Importantly these LDs appeared indistinguishable from TAG/SE-containing LDs [31]. Plant LDs (known as oil bodies or oleosomes) store large amounts of wax esters (i.e. esters of a fatty acid and a fatty alcohol) [32]. Furthermore, a variety of other hydrophobic molecules have been found to accumulate in LDs. These include $O$-acyl ceramide [33], squalene, vitamin E and long-chain polyprenols. Thus, multiple structurally diverse molecules are stored in LDs, however whether they are capable of triggering LD formation is unknown.

Newly synthesized neutral lipids, due to their hydrophobicity, are thought to disperse between the leaflets of the ER bilayer. Upon reaching a critical concentration, neutral lipids nucleate (i.e. phase-separate and coalesce) [34], forming lens-like structures (Figure 1A, I). Decreased interaction with other membrane components, such as phospholipids and proteins, appears to favor the demixing of neutral lipids into a lens [34]. Whereas the transient nature of early LD biogenesis intermediates complicates its characterization in vivo, neutral lipid nucleation has been assessed in silicio and in vitro. In model membranes, TAG coalesces into lenses at concentrations in the range of 5-10 mol\% $[35,36]$. In yeast cells, where LD biogenesis can be triggered synchronously, lens- 
like structures of approx. $50 \mathrm{~nm}$ diameter were observed by EM tomography soon after inducing TAG biosynthesis [37], providing the first in vivo evidence for the "lens model" of LD biogenesis.

Small, newly formed neutral lipid lenses are thought to fuse into bigger and more stable lenses (Figure 1A, II). Fusion of multiple smaller lenses to a singular, larger lens reduces the surface-to-volume ratio, providing more efficient sequestering of the neutral lipids between the ER leaflets. Indeed, recent molecular dynamics simulations show rapid diffusion of TAG lenses within the bilayer resulting in their eventual coalescence into larger lens [38]. Persistent neutral lipid synthesis likely also contributes to lens growth in vivo.

A large, spherical neutral lipid lens could be hosted permanently between the ER leaflets. However, in vivo, neutral lipid lenses move from the membrane equator towards the cytosol, a process described as directional budding (Figure 1A, III). During budding, the cytoplasmic leaflet of the ER membrane is thought to provide the phospholipids forming the monolayer of the nascent LD. Budding is also accompanied with further LD expansion requiring continuous phospholipid supply. Increasing evidence suggests that budding occurs at defined ER domains [39], which may have distinct protein and lipid composition compared to the bulk ER membranes. The (local) lipid composition dictates the membrane physical properties [40-42], which can be modulated by various proteins implicated in facilitating LD biogenesis .

Recent experimental and theoretical studies suggest that membrane intrinsic curvature (conferred by phospholipid shapes) influences LD budding (Figure 1B). According to the shape-structure concept of lipid polymorphism [43], cylindrical-shaped lipids, i.e. molecules with head group cross-sectional area similar to that of the acyl chains, assemble stable bilayers. In contrast, non-bilayer phospholipids, with a head-group cross-section larger than that of the acyl chain region (inverted cone-shaped lipids, type I lipids) or with the converse geometry (cone shape; type II lipids) tend to form structures with positive curvature (such as micelles) or negative curvature (hexagonal phases), respectively (Figure 1B). Due to their non-bilayer propensity, high concentration of these lipids in a planar lipid bilayer leads to curvature stress, which could be seen as the stored energy in the two 'frustrated' leaflets of the bilayer. Thus, high concentration of type I lipids in bilayers favor positively curved membranes, whereas type II lipids favor negative curvature. Lipid analysis of LDs showed that they are enriched in the cylindrical lipids PC and PI, and have lower amounts of cone-shaped PE [44]. No data on inverted cone-shaped lipids was presented in this study. In silico data suggests that local distribution of PLs at sites of LD formation influence LD budding and affect budding directionality [45]. Type I lipids such as lyso-PC, were found to favor LD budding, whereas type II lipids such as diunsatured-PE and DAG, were found to impair LD budding [45]. These observations are in line with type I lipids favouring positive curved structures, such as the curved LD surface, and type II lipids disfavouring these structures.

A second critical membrane parameter during LD budding is surface tension, which is defined as the energetic cost of exposing hydrophobic LD core to the aqueous surroundings [46]. Conditions of very low or very high surface tension, corresponding to dense or sparse phospholipid coverage of the neutral lipid lens respectively, disfavor directional LD budding. In particular, budding is disfavored when the surface tensions on 
both halves of the lens match. On the contrary, LD budding is favored by imbalances in surface tension between the two leaflets of the ER membrane, as shown recently [47], and may be sufficient to impose directionality to LD budding [48]. Recent in vitro and in vivo work suggest that increased availability of PLs on the cytoplasmic leaflet lowers the surface tension of this membrane leaflet, thus promoting the budding of LDs towards the cytosol $[46,48]$. A reduction in surface tension of the cytosolic leaflet may also be achieved by insertion of proteins, such as perilipins (see below) as a consequence of increasing the overall surface area of the cytoplasmic leaflet [47].

LD budding is accompanied with the recruitment of several LD-specific proteins (Figure 1C), which either diffuse from the ER to the LD monolayer (Class I proteins) or are recruited directly from the cytosol (Class II proteins, such as perilipins; see below) [49]. Class I proteins behave as integral membrane proteins but lack luminal domains. Their membrane association is typically mediated by a hydrophobic hairpin, a domain that can be accommodated both in the ER bilayer and the LD monolayer [49]. For Class II proteins, LD association often involves an amphipathic helix but other motifs, such as protein acylation (providing a lipidic anchor) have also been described [50]. In addition, proteinprotein interactions can also recruit cytosolic proteins to the LD surface.

After biogenesis, many LDs remain in close contact with the ER. However, a fraction of LDs releases from the ER, via a yet unknown mechanism. Interestingly, detached LDs can associate with the ER and "dock" onto the ER membrane, showing that this process is reversible. Re-attachment of LDs to the ER was shown to be dependent on the COPI coatamer complex [51-53].

\section{Proteins involved in LD formation}

\subsection{Lipid biosynthetic enzymes}

3.1.1. Neutral lipid biosynthetic enzymes

The most abundant neutral lipids SE and TAG are synthesized by acylation of their membrane lipid precursors, sterols (ST) and diacylglycerol (DAG,) respectively (Figure 2A). Sterol esters are mainly formed by steryl $O$-acyltransferase enzymes (SATs) such as Are1 and Are2 in yeast [54] and SOAT1 and SOAT2 in mammals. Mammalian cells also have a transacylase enzyme, LCAT1, that transfers an acyl chain from PC to cholesterol (lechitin:cholesterol acyl transferase).

TAG is formed by acyl-CoA dependent acylation of the free hydroxyl group of DAG, catalyzed by DAG $O$-acyltransferases (DGATs); Dga1 in yeast, and DGAT1 and DGAT2 in mammals. The mammalian DGATs differ in topology, with DGAT1 having its active site in the ER lumen, whereas the active site of DGAT2 faces the cytosol, similar to yeast Dga1. Thus, TAG produced on either side of the ER membrane can be packaged into LDs.

Interestingly, yeast Lro1 also synthesizes TAG and has its catalytic site on the ER lumen [55]. Lro1 has sequence similarity to the mammalian LCAT [56], also using phospholipids as an acyl chain donor in a phospholipid:DAG transacylase reaction. Lro1 appears to have a small contribution to TAG synthesis but curiously, in stationary phase, it relocalizes to the inner nuclear membrane and contributes to the formation of nuclear lipid droplets [57].

\subsubsection{Synthesis of neutral lipid precursors}


The TAG precursor DAG is derived from the general glycerolipid precursor phosphatidic acid (PA), formed by sequential acylation of glycerol-3-phosphate by G3P-acyltransferase (GPAT) and acyl-G3P-acyltransferases (AGPAT) enzymes (Figure 2A, see also [58]). Interestingly, while different GPATs and AGPATs have a certain degree of redundancy in vivo, they have unique functions and facilitate metabolic channelling of distinct pools of PA either to the synthesis of membrane glycerophospholipids or neutral lipids [59]. For example, yeast has two GPAT homologs (Gpt2/Gat1 and Sct1/Gat2) and their combined loss is lethal, indicating functional redundancy. However, Gpt2 appears to preferentially channel lipids to TAG formation. In oleate-supplemented media, gpt2 $\Delta$ mutants show fewer LDs and reduced viability, while Gpt2 overexpression increases LD size [60].

The hydrolysis of PA to DAG by the PA-hydrolase (PAH), Pah1 in yeast and Lipin in mammals, is a key decision point between phospholipid or TAG biosynthesis, leading respectively to membrane expansion or lipid storage [61,62]. PAH activity is tightly controlled via phosphorylation [63] and acetylation [64], serving as an axis for the regulation of cellular DAG levels. Yeast cells devoid of the main PAH enzyme Pah1 are strongly decreased in LDs and show expanded ER [65], indicating that lipid storage via LD biosynthesis may serve as a regulatory mechanism for organelle size and membrane surface area in general.

Activated fatty acids (acyl-coenzyme A; acyl-CoA) are used as acyl chain donor in the synthesis of TAG and SE by DGATs and SOATs. Acyl-CoA can be synthesized de novo from acetyl-CoA by the fatty acid synthase (FAS) complex, or by activation of free fatty acids (FFAs) by fatty acid activating (FAA) enzymes. Recently, yeast Mdm1, which tethers LDs to the nuclear-vacuole contact site, was shown to co-immunoprecipitate with the FAA enzyme Faa1 and FAS subunits Fas1 and Fas2, suggesting local production of acyl-CoA at sites of LD biogenesis [66].

\subsection{Seipin}

\subsubsection{Seipin functions in LD biogenesis, maintenance and dynamics}

Seipin was first identified as the product of the BSCL2 gene, which is mutated in patients with Berardinelli-Seip congenital lipodystrophy [67]. Seipin is an evolutionarily conserved ER integral membrane protein, with two transmembrane helices and a large ER lumenal domain. Yeast Seipin, known as Fld1 (ㅌew Lipid Droplets) or Sei1 was identified in genetic screens for mutants with aberrant LD morphology $[68,69]$. In these mutant cells, LDs are highly heterogeneous, present either in clusters of tiny droplets or in very few numbers of supersized LDs. Depletion of Seipin in fly and human cells results in similarly aberrant and heterogeneous LD morphologies suggesting a general role of Seipin in LD formation. In all cell types analyzed so far, Seipin is observed in foci along the ER membrane, many of which localize at contact sites with LDs (Figure 2B) [70-73]. Consistent with an important role at the interface between the two organelles, EMtomography showed aberrant ER-LD contacts in Seipin deficient cells. Likely as a consequence of these aberrant contacts, induction of LD formation in Seipin-deficient yeast or cultured mammalian cells results in slower neutral lipid accumulation in newly synthesized LDs. In addition, Seipin mutants have aberrant LD proteomes suggesting that its function also affects LD protein targeting [71,72]. These observations point to a major role of Seipin in controlling the partitioning of both lipids and proteins into LDs. 
Besides stabilizing ER-LD contacts, Seipin appears to determine the sites of LD formation. Stimulation of LD formation upon artificial immobilization of Seipin at the nuclear envelope, i.e. an ER domain where Seipin does not normally concentrate, leads to accumulation of LDs at the nuclear envelope [74]. In addition, Seipin appears to have important roles after biogenesis, influencing the maintenance of LD function and morphology. Using an ingenious assay to induce Seipin degradation, it was recently shown that acute Seipin depletion triggers defects in LD morphology, with a reduction in the number of small LDs and concomitant increase in supersized LDs [75]. A combination of in vivo and in vitro experiments, as well as simulations in silico, suggest that these changes are triggered by a phenomenon of LD ripening, in which neutral lipids diffuse from smaller to larger LDs using the ER bilayer as a conduit [75]. In this scenario, Seipin would facilitate the flow of neutral lipids from the ER to LDs while preventing the flux in the opposite direction, possibly acting as a "valve".

Interstingly, while most organisms contain a single Seipin gene, Arabidopsis thalliana has three Seipin paralogs that are non redundant in function. [76]. LD localization and their morphology is impaired upon loss of function of one of the three Seipin paralogs, thus all three are essential for the formation of proper LDs [77]. How these three Seipin homologs control LD biogenesis, morphology and localization is yet unclear. One might surmise that all three homologs are required to form a functional Seipin hetero-oligomer, though another possibility is that other factors in plants are required for correct LD formation. For a more detailed review of plants Seipins, the reader is referred to [78].

\subsubsection{Is Seipin a lipid-binding protein?}

Seipin consists of two transmembrane helices very proximal to the N and C-termini with an extended ER luminal domain. While expression of this core region of Seipin is sufficient for normal LD formation in cultured cells, mammalian Seipin has an extended C-terminal cytoplasmic domain, of yet unknown function [69]. Earlier biochemical and biophysical studies suggested that Seipin oligomerizes $[79,80]$, an observation that was confirmed by the recently solved structures of human [81] and fly [82] Seipin. The high resolution Seipin structures revealed that these proteins assemble into a ring-like structure of 11- and 12- protomer subunits, respectively (Figure 3A). Irrespective of the number of protomers, Seipin oligomerization is mediated through interactions within the ER luminal domain. Mutations preventing oligomer assembly showed defective LD morphology indicating that Seipin higher-order assemblies are required for its function in LD formation. In the individual Seipin protomers, the extended ER luminal domain adopts an eight $\beta$-strand sandwich fold capped on the inner surface of the ring by two short $\alpha$-helical elements (Figure 3B). The ring formed by the luminal $\beta$-sandwiches appears to sit parallel to the membrane (Figure 3A). In contrast, the $\alpha$-helices, which are rich in hydrophobic residues, project inwards, suggesting that they are buried in the luminal leaflet of the ER membrane. In human seipin, this hydrophobic helix is necessary for the interaction with promethin/LDAF1, which appears to form a stoichiometric complex with seipin [83] (see below). Interestingly, the Seipin $\beta$-strand sandwich shows remarkable similarity with $\beta$-sandwiches present in lipid-binding proteins (Figure 3B), such as the Niemann-Pick disease type $C$ protein 2 (NPC2), which binds to sterols, and the $\mathrm{C} 2$ domain of protein kinase $\mathrm{C}$ (PKC), which binds phosphatidylserine. In vitro lipidbinding experiments suggest that Seipin's $\beta$-sandwich can bind anionic phospholipids, such as PA [81]. However, whether Seipin has lipid-binding activity in vivo, the nature of a potential lipid ligand, and how lipid-binding affects Seipin's ability to promote LD formation are important open questions in the field. 


\subsubsection{Seipin interacting proteins}

While its potential lipid-binding activity awaits further characterization, Seipin was shown to bind various proteins. In yeast, the function of the Seipin Fld1 requires its binding to Ldb16, an ER protein consisting of two transmembrane domains and an extended cytosolic C-terminal region [72]. Yeast cells lacking Ldb16 have normal levels of the Seipin Fld1 however display aberrant LDs, indistinguishable from fld1 $1 \Delta$ mutants. These observations indicate that in yeast, Seipin function is carried out by a Fld1/Ldb16 complex. Curiously, expression of human Seipin in yeast cells lacking either Fld1, Ldb16 or both restore normal LD morphology showing that requirement for Ldb16 is yeastspecific [72]. The nature of this difference is not yet clear, but it is consistent with the absence of Ldb16 homologs in higher eukaryotes.

A proteomics screen for binding partners of the yeast Fld1/Ldb16 complex identified Ldo16 and Ldo45 proteins (Lipid Droplet Organization protein of $16 \mathrm{kDa}$ and $45 \mathrm{kDa}$, respectively) $[84,85]$. Interestingly, the two proteins are encoded by the same gene that can yield a long and a short transcripts giving rise to Ldo45 and Ldo16, respectively. In contrast to Fld1 and Ldb16 mutants, cells lacking Ldo16 and Ldo45 have much more subtle defects in LD morphology suggesting that these proteins act as regulators of the core seipin function [85]. Ldo16 and Ldo45 proteins associate with the ER membrane through two transmembrane segments but appear to have distinct effects on the Fld1/Ldb16 complex. While Ldo45 favors LD growth and TAG accumulation, Ldo16 appears to function primarily during LD consumption through lipophagy. Furthermore, the relative abundance of the two proteins varies according to cellular metabolism [85]. While the levels of Ldo16 are constant, Ldo45 levels are high during nutrient-rich periods, prone to LD formation and decrease steadily as nutrients become scarce. Besides binding to Fld1/Ldb16 complex in the ER, Ldo16 and Ldo45 proteins also distribute to the surface of LDs. Curiously, they localize preferentially to LDs proximal to a membrane contact site between the nuclear envelope-ER and the vacuole (the nuclear-vacuolar junction; NVJ) [83].

Iterative homology sequence searches revealed the similarity between Ldo45 and a conserved family of ER membrane proteins defined by human Promethin [84] (also known as TMEM159, recently named Lipid Droplet Assembly Factor 1; LDAF1 [86]). A number of other observations suggest that Promethin/LDAF1 and Ldo45 are indeed functional homologues. Consistent with high Ldo45 levels during lipogenesis in yeast, Promethin/LDAF1 expression increases during lipid storage in both mouse liver and adipose tissue [83]. Furthermore, Promethin/LDAF1 co-precipitates with Seipin $[83,86]$ forming a near stoichiometric complex [86]. Like in yeast, loss of Promethin/LDAF1 or expression of a Seipin mutant defective in Promethin/LDAF1 binding leads to only mild effects in LD morphology. How Promethin/LDAF1 regulates LD formation is unclear, but it was proposed that it stabilizes Seipin at sites of LD assembly that coincide with TAG lenses. Expansion of the lense would disrupt the Seipin-Promethin/LDAF1 interaction, leading to Promethin/LDAF1 to accumulate at the LD surface. Curiously, LDAF1 is also sequence-related to oleosins, which are key structural LD proteins in plants [87], suggesting that these proteins may regulate LD behaviour through multiple means.

\subsubsection{Seipin as a regulator of lipid metabolism?}


Seipin has been shown to interact with various lipid biosynthetic enzymes, including acyl-CoA desaturase [88], GPAT [89], AGPAT [90] and PAH enzymes [90,91]. For example, GPAT3 in mammals and the yeast homologues Sct1 and Gpt2 interact with Seipin in mammalian and yeast cells, respectively [89]. Enzyme activity measurements showed that GPAT activity had an inverse correlation with the presence of Seipin, suggesting that it may act as a negative regulator of GPAT activity. Interaction of Seipin with lipid metabolic enzymes could provide an axis to regulate lipid biosynthesis, but in most cases the relevance of the interactions remained unexplored.

Interestingly, very recent work showed that the yeast Fld1/Ldb16 seipin complex interacts with the serine-palmitoyltransferase (SPT) complex, which catalyzes the ratelimiting step in sphingolipid biosynthesis [92]. Sphingolipids are a class of membrane lipids structurally distinct from phospholipids and most abundant in the Golgi system and plasma membrane [93]. Like most membrane lipids, sphingolipids are synthesized in the ER but opposed to PLs and sterols, sphingolipids are not converted to storage lipids in bulk amounts. The Seipin-SPT interaction occurs specifically at the ER-LD contacts, and in presence of high sphingolipid levels, resulting in local inhibition of SPT activity [92]. Curiously, SPT inhibition by Seipin was shown to be independent of the well established SPT regulators Orm $1 / 2$ and Tsc13. In addition to SPT, Seipin was shown to also inhibit FA elongase activity [92]. While sphingolipids are not involved in LD formation, local SPT inhibition by Seipin could be important to prevent local accumulation of sphingolipid intermediates that may interfere with LD biogenesis. Alternatively, inhibition of SPT and FA elongation reduces the local metabolic draw on acyl-CoA (mainly palmitoyl-CoA), which could influence local TAG/SE synthesis and LD formation.

\subsection{Perilipin}

Perilipins (PLINs) are a major family of LD proteins that are thought to act as LD coat proteins (Figure 2B). PLINs are defined by the presence of a PAT domain, usually also including a predicted 4-helix bundle and an amphipathic helix (AH) [94]. Mammals have five different perilipins (PLIN1-5), flies have two (Lsd1 and LSD2) whereas in yeast only one PLIN has been identified (Pet10/Pln1). The 5 mammalian PLINs have distinct expression patterns, with Perilipins 2 and 3 being the most ubiquitous. While most Perilipins bind to LDs by interacting with monolayer phospholipids, Perilipin 4, with an unusually long amphipathic helix, stabilizes LDs by binding directly to neutral lipids [95]. The various PLINs also bind to distinct LD populations [96,97][97], likely reflecting functional diversity. For example in preadipocytes, PLIN2 coats LDs, whereas after differentiation into mature adipocytes, PLIN2 is replaced by PLIN1 as LD size increases. PLIN1 coating inhibits lipolysis by preventing the binding of lipases, facilitating the formation of giant LDs in white adipose tissue [96].

Perilipin 3 was shown to play a role in LD biogenesis [98]. In cultured mammalian cells, upon stimulation of LD formation, TIP47/PLIN3 is quickly recruited to ER sites involved in LD assembly. In addition, depletion of TIP47/PLIN3 reduces TAG incorporation into nascent LDs resulting in less and smaller LDs. Similarly, yeast Pet10/Pln1 is recruited to nascent LDs, promotes TAG accumulation and LD stability [98]. While the precise mechanism by which perilipins promote LD formation requires further investigation, recent in vitro work suggests that perilipin recruitment to nascent LDs increases membrane area resulting in reduced membrane surface tension and thereby more efficient LD budding from the ER [47]. 


\subsection{FIT proteins}

The Fat-Induced Transcript 2 (FIT2) protein is the founding member of an evolutionarily conserved family of ER membrane proteins. FIT2 proteins are essential for the viability of mice and worms [37,99], and FIT2-depletion in cells leads to aberrant LDs. In all cell types investigated so far, including human, mouse, worm and yeast, loss of FIT2 results in neutral lipid lens embedded in the ER membrane, suggesting that FIT2 is required for LD budding towards the cytosol [37]. However, there is no unified model for the function of FIT2 during LD budding yet. Besides the LD budding defect, FIT2-deficient yeast cells display slightly higher ER levels of DAG. As non-bilayer lipids disfavor LD budding, the DAG accumulation may explain the budding defect observed in FIT mutants [45]. In an assay following synchronous LD formation in yeast, both FIT2-like proteins, Scs3 and Yft2, appear to concentrate transiently at sites of LD biogenesis. These observations led to the suggestion that FIT2 proteins may promote LD budding by controlling DAG levels at LD biogenesis sites. This possibility is also consistent with earlier experiments showing that purified human FIT2 binds both TAG and DAG in vitro [100].

While these data suggest a role of FIT proteins at LD biogenesis sites other observations point towards a broader role of FIT2 proteins in ER phospholipid homeostasis. For example, scs3 3 mutant was identified in a screen for genes with altered phospholipid metabolism and genetically interacts with various genes involved in phospholipid biosynthesis [101]. In agreement with a general role in lipid metabolism, a recent preprint reports that FIT2 depletion in cultured mammalian cells treated with oleate resulted in decreased synthesis of TAG and most phospholipids, with the exception of phosphatidic acid, which was highly increased [102]. The lipid changes resulted in altered ER morphology, with the appearance of membrane whorls. Similar structures were detected in $\operatorname{scs} 3 \Delta$ and $\operatorname{scs} 3 \Delta y f t 2 \Delta$ mutants in yeast [103]. In contrast to yeast cells, stimulation of LD formation in mammalian cultured cells with oleate supplementation did not result in FIT2 relocalization, which remained uniformly distributed throughout the ER. Thus, it remains unresolved whether FIT2 contributes to LD formation by acting locally or whether the LDs defects are a consequence of a broader role in ER lipid homeostasis.

Recently, analysis of FIT2 proteins amino acid sequences revealed that these proteins are related to lipid phosphatase/phosphotransferase (LPT) enzymes, including the residues in the putative catalytic site, suggesting they may act as enzymes [103]. Mutations of these putative catalytic residues in Scs3 and Yft2 behaved as null mutants further supporting the possible enzymatic functions of FIT2 proteins. Purified human wild-type FIT2, but not a putative catalytic site mutant, hydrolyzed PA and lysophosphatidic acid to DAG and monoacylglycerol, respectively, in vitro [102]. How robust is this activity among the various FIT2 proteins and whether in cells FIT2 family members act as enzymes are important open questions. According to topology predictions for human FIT2, which indicate that the potentially catalytic residues lie at the interface between the inner ER membrane leaflet and the ER lumen, and the activity observed in vitro, FIT2 may generate DAG (and/or monoacylglycerol) on the inner leaflet of the ER [102]. Given the high rate of spontaneous transbilayer movement of DAG, one may speculate that DAG generated on the inner leaflet of the ER can be converted into phospholipids in the outer leaflet. Such a process would result in an asymmetric membrane, with lower surface tension on the cytosolic leaflet, a condition shown in biophysical studies to promote LD budding. 
This scenario, compatible with the absence of LD budding in FIT2 mutants, should be directly tested in the future.

\subsection{Pex30}

Pex30 is the founding member of a family ER membrane proteins in yeast defined by the presence of a membrane shaping reticulon-homology domain and a dysferlin-like domain of unknown function. The Pex30-like family also includes Pex28, Pex29, Pex31 and Pex32, all of which have been implicated in the regulation of the size and shape of peroxisomes $[104,105]$. In the case of Pex30 and Pex31, this is likely achieved through the reticulonhomology domain, which by stabilizing high membrane curvature at specific ER sites, facilitates the budding of pre-peroxisomal vesicles that eventually give rise to peroxisomes [106].

Surprisingly, Pex30 also concentrates at ER sites involved in LD formation revealing potential parallels between the biogenesis of peroxisomes and LDs $[107,108]$. Pex30 contribution to LD biogenesis becomes more apparent in the absence of Seipin, a condition that leads to dramatic Pex30 accumulation in ER regions adjacent to LDs. In addition, simultaneous depletion of Seipin and Pex30 results in severe inhibition of LD biogenesis accompanied by ER morphology defects and altered lipid homeostasis. How Pex30 contributes to LD formation is unclear but correction of phospholipid imbalances reestablished to some extent LD formation in cells lacking Pex30 and Seipin [107]. This suggests that Pex30 contributes to maintaining phospholipid homeostasis during LD formation. Based on similarity at the reticulon-homology domain, MCTP2 was proposed to be the functional homolog of Pex30 in mammals. Consistently, MCTP2 localizes both to sites of LD and pre peroxisomal vesicles biogenesis and its depletion resulted in aberrant LD formation in cultured cells as well as in worms [108]. While the membrane shaping properties of the RHD of Pex30 and MCTP2 appear to play an important role in organelle budding, how these proteins affect lipid homeostasis specifically during LD biogenesis should be further investigated.

\subsection{Nexin family proteins}

MDM1 was originally identified in a yeast screen for mutants with mitochondrial distribution and morphology defects [109]. Recently it was shown that Mdm1, as well as its human ortholog SNX14, are ER-resident proteins that bind LDs [110,111]. In particular, Mdm1 concentrates specifically at LDs proximal to the NVJ. In fact, Mdm1 overexpression is sufficient to trigger LD formation at the NVJ [110], suggesting that Mdm1 is important in the spatial organization of lipid storage [5]. Curiously, the first 51 $\mathrm{N}$-terminal amino acids of Mdm1 encompassing the ER membrane domain, also include the determinant for localization to LDs proximal to the NVJ [112]. Mdm1 mutants show defects in ER morphology and TAG accumulation, resulting in increased susceptibility to lipotoxicity [112]. Similar LD morphology defects have been observed in cultured cells upon SNX14 depletion. Conversely, SNX14 overexpression results in more LDs [111]. Characterization of Mdm1/SNX14 homolog in Drosophila melanogaster, known as Snazarus, further supports a general role of this protein family in the compartmentalization of lipid storage. Snazarus is critical to maintaining a population of LDs to the cell periphery and its overproduction leads to expansion of the peripheral LDs resulting in flies with increased resistance to starvation and lifespan [12]. While clearly 
important for the spatial organization of lipid metabolism across various cell types and cellular contexts, the role of Mdm1/SNX14 protein family in LD biogenesis remains less clear.

\section{Concluding remarks}

Over the last several years, there were significant advances in understanding LD biology. Cutting edge light and electron microscopy revealed the complex pattern of LD distributions and interactions within cells. Proteomics and genetic screens identified a list of factors affecting LD biogenesis and behaviour. Biophysical and theoretical approaches started to define the physical principles underlying LD formation, while structural biology started to provide molecular insights about the proteins involved in the process. The emerging picture indicates that LD biogenesis occurs at specific ER domains, marked by the presence of various proteins and perhaps of a lipid composition distinct from the surrounding ER. It has become clear that neutral and phospholipids alone can drive LD formation, but in vivo this process appears to be highly orchestrated by a growing list of proteins. Thus, a major challenge for the field will be to define the precise molecular function of these components and how they integrate metabolic requirements for appropriate LD function in lipid and energy homeostasis. 


\section{Acknowledgements}

We thank Joana Ferreira, Sonia Muliyil, and Daniel Fonseca for comments on the manuscript and current and former members of the Carvalho lab for fruitful discussions. We apologize to colleagues whose works were not cited due to size limitations. P.C. is financially supported by a Welcome Trust Investigator Award (202642/Z/16/Z) and a BBSRC grant (BB/R018375/1).

\section{References}

[1] K. Tauchi-Sato, S. Ozeki, T. Houjou, R. Taguchi, T. Fujimoto, The surface of lipid droplets is a phospholipid monolayer with a unique fatty acid composition, J. Biol. Chem. 277 (2002) 44507-44512. doi:10.1074/jbc.M207712200.

[2] T. Fujimoto, R.G. Parton, Not just fat: the structure and function of the lipid droplet. Cold Spring Harb Perspect Biol 3:1-17, Cold Spring Harb Prespectives Biol. 3 (2011) 1-17.

[3] J.A. Olzmann, P. Carvalho, Dynamics and functions of lipid droplets, Nat. Rev. Mol. Cell Biol. 20 (2019) 137-155. doi:10.1038/s41580-018-0085-z.

[4] T.C. Walther, J. Chung, R. V. Farese, Lipid Droplet Biogenesis, Annu. Rev. Cell Dev. Biol. 33 (2017) 491-510. doi:10.1146/annurev-cellbio-100616-060608.

[5] M. Henne, J.M. Goodman, H. Hariri, Spatial compartmentalization of lipid droplet biogenesis, Biochim. Biophys. Acta - Mol. Cell Biol. Lipids. (2019). doi:10.1016/j.bbalip.2019.07.008.

[6] M. Schuldiner, M. Bohnert, A different kind of love - lipid droplet contact sites, Biochim. Biophys. Acta - Mol. Cell Biol. Lipids. 1862 (2017) 1188-1196. doi:10.1016/j.bbalip.2017.06.005.

[7] A.M. Valm, S. Cohen, W.R. Legant, J. Melunis, U. Hershberg, E. Wait, A.R. Cohen, M.W. Davidson, E. Betzig, J. Lippincott-Schwartz, Applying systems-level spectral imaging and analysis to reveal the organelle interactome, Nature. 546 (2017) 162167. doi:10.1038/nature22369.

[8] N. Shai, E. Yifrach, C.W.T. Van Roermund, N. Cohen, C. Bibi, L. Ijlst, L. Cavellini, J. Meurisse, R. Schuster, L. Zada, M.C. Mari, F.M. Reggiori, A.L. Hughes, M. EscobarHenriques, M.M. Cohen, H.R. Waterham, R.J.A. Wanders, M. Schuldiner, E. Zalckvar, Systematic mapping of contact sites reveals tethers and a function for the peroxisome-mitochondria contact, Nat. Commun. 9 (2018). doi:10.1038/s41467018-03957-8.

[9] A. Herms, M. Bosch, B.J.N. Reddy, N.L. Schieber, A. Fajardo, C. Rupérez, A. Fernández-Vidal, C. Ferguson, C. Rentero, F. Tebar, C. Enrich, R.G. Parton, S.P. Gross, A. Pol, AMPK activation promotes lipid droplet dispersion on detyrosinated microtubules to increase mitochondrial fatty acid oxidation, Nat. Commun. 6 (2015) 7176. doi:10.1038/ncomms8176.

[10] J.P. Layerenza, P. González, M.M. García De Bravo, M.P. Polo, M.S. Sisti, A. VesLosada, Nuclear lipid droplets: A novel nuclear domain, Biochim. Biophys. Acta Mol. Cell Biol. Lipids. 1831 (2013) 327-340. doi:10.1016/j.bbalip.2012.10.005.

[11] R. Uzbekov, P. Roingeard, Nuclear lipid droplets identified by electron microscopy of serial sections, BMC Res. Notes. 6 (2013) 2-5. doi:10.1186/1756-0500-6-386.

[12] R. Ugrankar, J. Bowerman, H. Hariri, M. Chandra, K. Chen, M.-F. Bossanyi, S. Datta, S. Rogers, K.M. Eckert, G. Vale, A. Victoria, J. Fresquez, J.G. McDonald, S. Jean, B.M. Collins, W.M. Henne, Drosophila Snazarus Regulates a Lipid Droplet Population at Plasma Membrane-Droplet Contacts in Adipocytes, Dev. Cell. 50 (2019) 557572.e5. doi:10.1016/j.devcel.2019.07.021. 
[13] S. Cermelli, Y. Guo, S.P. Gross, M.A. Welte, The Lipid-Droplet Proteome Reveals that Droplets Are a Protein-Storage Depot, Curr. Biol. 16 (2006) 1783-1795. doi:10.1016/j.cub.2006.07.062.

[14] P. Anand, S. Cermelli, Z. Li, A. Kassan, M. Bosch, R. Sigua, L. Huang, A.J. Ouellette, A. Pol, M.A. Welte, S.P. Gross, A novel role for lipid droplets in the organismal antibacterial response, Elife. 1 (2012). doi:10.7554/eLife.00003.

[15] Y. Jo, I.Z. Hartman, R.A. DeBose-Boyd, Ancient ubiquitous protein-1 mediates sterol-induced ubiquitination of 3-hydroxy-3-methylglutaryl CoA reductase in lipid droplet-associated endoplasmic reticulum membranes., Mol. Biol. Cell. 24 (2013) 169-83. doi:10.1091/mbc.E12-07-0564.

[16] I.Z. Hartman, P. Liu, J.K. Zehmer, K. Luby-Phelps, Y. Jo, R.G.W. Anderson, R.A. DeBose-Boyd, Sterol-induced dislocation of 3-hydroxy-3-methylglutaryl coenzyme A reductase from endoplasmic reticulum membranes into the cytosol through a subcellular compartment resembling lipid droplets., J. Biol. Chem. 285 (2010) 19288-98. doi:10.1074/jbc.M110.134213.

[17] Y. Ohsaki, J. Cheng, M. Suzuki, A. Fujita, T. Fujimoto, Lipid droplets are arrested in the ER membrane by tight binding of lipidated apolipoprotein B-100., J. Cell Sci. 121 (2008) 2415-22. doi:10.1242/jcs.025452.

[18] M. Suzuki, T. Otsuka, Y. Ohsaki, J. Cheng, T. Taniguchi, H. Hashimoto, H. Taniguchi, T. Fujimoto, Derlin-1 and UBXD8 are engaged in dislocation and degradation of lipidated ApoB-100 at lipid droplets., Mol. Biol. Cell. 23 (2012) 800-10. doi:10.1091/mbc.E11-11-0950.

[19] D.J. Greenwood, M.S. Dos Santos, S. Huang, M.R.G. Russell, L.M. Collinson, J.I. MacRae, A. West, H. Jiang, M.G. Gutierrez, Subcellular antibiotic visualization reveals a dynamic drug reservoir in infected macrophages, Science. 364 (2019) 1279-1282. doi:10.1126/science.aat9689.

[20] T.C. Walther, R. V. Farese, Un-phased: Lipid Droplets Modulate the Bioavailability of Antibiotics, Dev. Cell. 50 (2019) 530-532. doi:10.1016/j.devcel.2019.08.009.

[21] J.B. Helms, D. V. Kaloyanova, J.R.P. Strating, J.J. van Hellemond, H.M. van der Schaar, A.G.M. Tielens, F.J.M. van Kuppeveld, J.F. Brouwers, Targeting of the Hydrophobic Metabolome by Pathogens, Traffic. 16 (2015) 439-460. doi:10.1111/tra.12280.

[22] N.L. Meyers, K.A. Fontaine, G.R. Kumar, M. Ott, Entangled in a membranous web: ER and lipid droplet reorganization during hepatitis $C$ virus infection, Curr. Opin. Cell Biol. 41 (2016) 117-124. doi:10.1016/j.ceb.2016.05.003.

[23] J. Zhang, Y. Lan, M.Y. Li, M.M. Lamers, M. Fusade-Boyer, E. Klemm, C. Thiele, J. Ashour, S. Sanyal, Flaviviruses Exploit the Lipid Droplet Protein AUP1 to Trigger Lipophagy and Drive Virus Production, Cell Host Microbe. 23 (2018) 819-831.e5. doi:10.1016/j.chom.2018.05.005.

[24] O. Laufman, J. Perrino, R. Andino, O. Laufman, J. Perrino, R. Andino, Viral Generated Inter-Organelle Contacts Redirect Lipid Flux for Genome Replication Article Viral Generated Inter-Organelle Contacts Redirect Lipid Flux for Genome Replication, Cell. 178 (2019) 275-289.e16. doi:10.1016/j.cell.2019.05.030.

[25] M. Graef, Lipid droplet-mediated lipid and protein homeostasis in budding yeast, FEBS Lett. 592 (2018) 1291-1303. doi:10.1002/1873-3468.12996.

[26] N. Krahmer, R. V. Farese, T.C. Walther, Balancing the fat: Lipid droplets and human disease, EMBO Mol. Med. 5 (2013) 905-915. doi:10.1002/emmm.201100671.

[27] T. Czabany, A. Wagner, D. Zweytick, K. Lohner, E. Leitner, E. Ingolic, G. Daum, Structural and biochemical properties of lipid particles from the yeast Saccharomyces cerevisiae, J. Biol. Chem. 283 (2008) 17065-17074. 
doi:10.1074/jbc.M800401200.

[28] M. Grzybek, A. Palladini, V.I. Alexaki, M.A. Surma, K. Simons, T. Chavakis, C. Klose, Ü. Coskun, Comprehensive and quantitative analysis of white and brown adipose tissue by shotgun lipidomics, Mol. Metab. 22 (2019) 12-20. doi:10.1016/j.molmet.2019.01.009.

[29] L. Sandager, M.H. Gustavsson, U. Ståhl, A. Dahlqvist, E. Wiberg, A. Banas, M. Lenman, H. Ronne, S. Stymne, Storage lipid synthesis is non-essential in yeast, J. Biol. Chem. 277 (2002) 6478-6482. doi:10.1074/jbc.M109109200.

[30] J. Petschnigg, H. Wolinski, D. Kolb, G. Zelling, C.F. Kurat, K. Natter, S.D. Kohlwein, Good fat, essential cellular requirements for triacylglycerol synthesis to maintain membrane homeostasis in yeast, J. Biol. Chem. 284 (2009) 30981-30993. doi:10.1074/jbc.M109.024752.

[31] M.R. Molenaar, T.A. Wassenaar, K.K. Yadav, Lecithin : Retinol Acyl Transferase ( LRAT ) induces the formation of lipid droplets, (2019) 1-38. doi:https://doi.org/10.1101/733931.

[32] M.L. Parker, G.J.P. Murphy, Oleosomes in flag leaves of wheat; their distribution, composition and fate during senesence and rust-infection, Planta. 152 (1981) 3643. doi:10.1007/BF00384982.

[33] C.E. Senkal, M.F. Salama, A.J. Snider, J.J. Allopenna, N.A. Rana, A. Koller, Y.A. Hannun, L.M. Obeid, Ceramide Is Metabolized to Acylceramide and Stored in Lipid Droplets, Cell Metab. 25 (2017) 686-697. doi:10.1016/j.cmet.2017.02.010.

[34] A.R. Thiam, L. Forêt, The physics of lipid droplet nucleation, growth and budding, Biochim. Biophys. Acta - Mol. Cell Biol. Lipids. 1861 (2016) 715-722. doi:10.1016/j.bbalip.2016.04.018.

[35] H. Khandelia, L. Duelund, K.I. Pakkanen, J.H. Ipsen, Triglyceride blisters in lipid bilayers: Implications for lipid droplet biogenesis and the mobile lipid signal in cancer cell membranes, PLoS One. 5 (2010) 1-8. doi:10.1371/journal.pone.0012811.

[36] L. Duelund, G.V. Jensen, H.K. Hannibal-Bach, C.S. Ejsing, J.S. Pedersen, K.I. Pakkanen, J.H. Ipsen, Composition, structure and properties of POPC-triolein mixtures. Evidence of triglyceride domains in phospholipid bilayers, Biochim. Biophys. Acta - Biomembr. 1828 (2013) 1909-1917. doi:10.1016/j.bbamem.2013.03.020.

[37] V. Choudhary, N. Ojha, A. Golden, W.A. Prinz, A conserved family of proteins facilitates nascent lipid droplet budding from the ER, J. Cell Biol. 211 (2015) 261271. doi:10.1083/jcb.201505067.

[38] V. Zoni, P. Campomanes, R. Khaddaj, A.R. Thiam, R. Schneiter, S. Vanni, Lipid droplet biogenesis is a liquid phase separation spatially regulated by seipin and membrane curvature, BioRxiv. (2019) 777466. doi:10.1101/777466.

[39] N.T. Nettebrock, M. Bohnert, Born this way - Biogenesis of lipid droplets from specialized ER subdomains, Biochim. Biophys. Acta - Mol. Cell Biol. Lipids. (2019). doi:10.1016/j.bbalip.2019.04.008.

[40] T. Harayama, H. Riezman, Understanding the diversity of membrane lipid composition, Nat. Rev. Mol. Cell Biol. 19 (2018) 281-296. doi:10.1038/nrm.2017.138.

[41] M.F. Renne, A.I.P.M. de Kroon, The role of phospholipid molecular species in determining the physical properties of yeast membranes, FEBS Lett. 592 (2018) 1330-1345. doi:10.1002/1873-3468.12944.

[42] G. Van Meer, D.R. Voelker, G.W. Feigenson, Membrane lipids: Where they are and how they behave, Nat. Rev. Mol. Cell Biol. 9 (2008) 112-124. 
doi:10.1038/nrm2330.

[43] P.R. Cullis, B. de Kruijff, Lipid polymorphism and the functional roles of lipids in biological membranes., Biochim. Biophys. Acta. 559 (1979) 399-420. doi:10.1016/0009-3084(86)90067-8.

[44] K. Grillitsch, M. Connerth, H. Köfeler, T.N. Arrey, B. Rietschel, B. Wagner, M. Karas, G. Daum, Lipid particles/droplets of the yeast Saccharomyces cerevisiae revisited: Lipidome meets Proteome, Biochim. Biophys. Acta - Mol. Cell Biol. Lipids. 1811 (2011) 1165-1175. doi:10.1016/j.bbalip.2011.07.015.

[45] V. Choudhary, G. Golani, A.S. Joshi, S. Cottier, R. Schneiter, W.A. Prinz, M.M. Kozlov, Architecture of Lipid Droplets in Endoplasmic Reticulum Is Determined by Phospholipid Intrinsic Curvature, Curr. Biol. 28 (2018) 915-926.e9. doi:10.1016/j.cub.2018.02.020.

[46] K. Ben M'barek, D. Ajjaji, A. Chorlay, S. Vanni, L. Forêt, A.R. Thiam, ER Membrane Phospholipids and Surface Tension Control Cellular Lipid Droplet Formation, Dev. Cell. 41 (2017) 591-604.e7. doi:10.1016/j.devcel.2017.05.012.

[47] A. Chorlay, L. Monticelli, J. Veríssimo Ferreira, K. Ben M’barek, D. Ajjaji, S. Wang, E. Johnson, R. Beck, M. Omrane, M. Beller, P. Carvalho, A. Rachid Thiam, Membrane Asymmetry Imposes Directionality on Lipid Droplet Emergence from the ER, Dev. Cell. 50 (2019) 25-42.e7. doi:10.1016/j.devcel.2019.05.003.

[48] A. Chorlay, A.R. Thiam, An Asymmetry in Monolayer Tension Regulates Lipid Droplet Budding Direction, Biophys. J. 114 (2018) 631-640. doi:10.1016/j.bpj.2017.12.014.

[49] N. Kory, R. V. Farese, T.C. Walther, Targeting Fat: Mechanisms of Protein Localization to Lipid Droplets, Trends Cell Biol. 26 (2016) 535-546. doi:10.1016/j.tcb.2016.02.007.

[50] K. Bersuker, C.W.H. Peterson, M. To, S.J. Sahl, V. Savikhin, E.A. Grossman, D.K. Nomura, J.A. Olzmann, A Proximity Labeling Strategy Provides Insights into the Composition and Dynamics of Lipid Droplet Proteomes, Dev. Cell. 44 (2018) 97112.e7. doi:10.1016/j.devcel.2017.11.020.

[51] F. Wilfling, H. Wang, J.T. Haas, N. Krahmer, T.J. Gould, A. Uchida, J.X. Cheng, M. Graham, R. Christiano, F. Fröhlich, X. Liu, K.K. Buhman, R.A. Coleman, J. Bewersdorf, R. V. Farese, T.C. Walther, Triacylglycerol synthesis enzymes mediate lipid droplet growth by relocalizing from the ER to lipid droplets, Dev. Cell. 24 (2013) 384-399. doi:10.1016/j.devcel.2013.01.013.

[52] A.R. Thiam, B. Antonny, J. Wang, J. Delacotte, F. Wilfling, T.C. Walther, R. Beck, J.E. Rothman, F. Pincet, COPI buds $60-\mathrm{nm}$ lipid droplets from reconstituted waterphospholipid- triacylglyceride interfaces, suggesting a tension clamp function, Proc. Natl. Acad. Sci. U. S. A. 110 (2013) 13244-13249. doi:10.1073/pnas.1307685110.

[53] F. Wilfling, A.R. Thiam, M.J. Olarte, J. Wang, R. Beck, T.J. Gould, E.S. Allgeyer, F. Pincet, J. Bewersdorf, R. V. Farese, T.C. Walther, Arf1/COPI machinery acts directly on lipid droplets and enables their connection to the ER for protein targeting, Elife. 2014 (2014) 1-20. doi:10.7554/eLife.01607.

[54] H. Yang, M. Bard, D.A. Bruner, A. Gleeson, R.J. Deckelbaum, G. Aljinovic, T.M. Pohl, R. Rothstein, S.L. Sturley, Sterol esterification in yeast: A two-gene process, Science (80-. ). 272 (1996) 1353-1356. doi:10.1126/science.272.5266.1353.

[55] V. Choudhary, N. Jacquier, R. Schneiter, The topology of the triacylglycerol synthesizing enzyme Lro1 indicates that neutral lipids can be produced within the luminal compartment of the endoplasmatic reticulum: Implications for the 
biogenesis of lipid droplets., Commun. Integr. Biol. 4 (2011) 781-4. doi:10.4161/cib.4.6.17830.

[56] P. Oelkers, A. Tinkelenberg, N. Erdeniz, D. Cromley, J.T. Billheimer, S.L. Sturley, A lecithin cholesterol acyltransferase-like gene mediates diacylglycerol esterification in yeast, J. Biol. Chem. 275 (2000) 15609-15612. doi:10.1074/jbc.C000144200.

[57] A.D. Barbosa, K. Lim, M. Mari, J.R. Edgar, L. Gal, P. Sterk, B.J. Jenkins, A. Koulman, D.B. Savage, M. Schuldiner, F. Reggiori, P.A. Wigge, S. Siniossoglou, Compartmentalized Synthesis of Triacylglycerol at the Inner Nuclear Membrane Regulates Nuclear Organization, Dev. Cell. (2019) 1-12. doi:10.1016/j.devcel.2019.07.009.

[58] J.C.M. Holthuis, A.K. Menon, Lipid landscapes and pipelines in membrane homeostasis, Nature. 510 (2014) 48-57. doi:10.1038/nature13474.

[59] J. Lee, N.D. Ridgway, Substrate channeling in the glycerol-3-phosphate pathway regulates the synthesis, storage and secretion of glycerolipids, Biochim. Biophys. Acta - Mol. Cell Biol. Lipids. (2019). doi:10.1016/j.bbalip.2019.03.010.

[60] N. Marr, J. Foglia, M. Terebiznik, K. Athenstaedt, V. Zaremberg, Controlling lipid fluxes at glycerol-3-phosphate acyltransferase step in yeast: Unique contribution of Gat1p to oleic acid-induced lipid particle formation, J. Biol. Chem. 287 (2012) 10251-10264. doi:10.1074/jbc.M111.314112.

[61] M. Carman, G, G. Han, Roles of phosphatidate phosphatase enzyme in lipid metabolism, Trends Biochem. Sci. $31 \quad$ (2006) 694-699. http://www.ncbi.nlm.nih.gov/entrez/query.fcgi?cmd=Retrieve\&db=PubMed\&do pt=Citation\&list_uids $=17079146$.

[62] J.M. Kwiatek, G.-S. Han, G.M. Carman, Phosphatidate-mediated regulation of lipid synthesis at the nuclear/endoplasmic reticulum membrane, Biochim. Biophys. Acta - Mol. Cell Biol. Lipids. (2019). doi:10.1016/j.bbalip.2019.03.006.

[63] G.M. Carman, G.S. Han, Phosphatidic acid phosphatase, a key enzyme in the regulation of lipid synthesis, J. Biol. Chem. 284 (2009) 2593-2597. doi:10.1074/jbc.R800059200.

[64] T.Y. Li, L. Song, Y. Sun, J. Li, C. Yi, S.M. Lam, D. Xu, L. Zhou, X. Li, Y. Yang, C.-S. Zhang, C. Xie, X. Huang, G. Shui, S.-Y. Lin, K. Reue, S.-C. Lin, Tip60-mediated lipin 1 acetylation and ER translocation determine triacylglycerol synthesis rate., Nat. Commun. 9 (2018) 1916. doi:10.1038/s41467-018-04363-w.

[65] O. Adeyo, P.J. Horn, S.K. Lee, D.D. Binns, A. Chandrahas, K.D. Chapman, J.M. Goodman, The yeast lipin orthologue Pah1p is important for biogenesis of lipid droplets, J. Cell Biol. 192 (2011) 1043-1055. doi:10.1083/jcb.201010111.

[66] H. Hariri, S. Rogers, R. Ugrankar, Y.L. Liu, J.R. Feathers, W.M. Henne, Lipid droplet biogenesis is spatially coordinated at ER-vacuole contacts under nutritional stress, EMBO Rep. (2017) e201744815. doi:10.15252/embr.201744815.

[67] J. Magré, M. Delépine, E. Khallouf, T. Gedde-Dahl, L. Van Maldergem, E. Sobel, J. Papp, M. Meier, A. Mégarbané, A. Bachy, A. Verloes, F.H. D’Abronzo, E. Seemanova, R. Assan, N. Baudic, C. Bourut, P. Czernichow, F. Huet, F. Grigorescu, M. de Kerdanet, D. Lacombe, P. Labrune, M. Lanza, H. Loret, F. Matsuda, J. Navarro, A. NivelonChevalier, M. Polak, J.J. Robert, P. Tric, N. Tubiana-Rufi, C. Vigouroux, J. Weissenbach, S. Savasta, J.A. Maassen, O. Trygstad, P. Bogalho, P. Freitas, J.L. Medina, F. Bonnicci, B.I. Joffe, G. Loyson, V.R. Panz, F.J. Raal, S. O’Rahilly, T. Stephenson, C.R. Kahn, M. Lathrop, J. Capeau, BSCL Working Group, Identification of the gene altered in Berardinelli-Seip congenital lipodystrophy on chromosome 11q13., Nat. Genet. 28 (2001) 365-70. doi:10.1038/ng585. 
[68] W. Fei, G. Shui, B. Gaeta, X. Du, L. Kuerschner, P. Li, A.J. Brown, M.R. Wenk, R.G. Parton, H. Yang, Fld1p, a functional homologue of human seipin, regulates the size of lipid droplets in yeast, J. Cell Biol. 180 (2008) 473-482. doi:10.1083/jcb.200711136.

[69] K.M. Szymanski, D. Binns, R. Bartz, N. V. Grishin, W.-P. Li, A.K. Agarwal, A. Garg, R.G.W. Anderson, J.M. Goodman, The lipodystrophy protein seipin is found at endoplasmic reticulum lipid droplet junctions and is important for droplet morphology, Proc. Natl. Acad. Sci. 104 (2007) 20890-20895. doi:10.1073/pnas.0704154104.

[70] H. Wang, M. Becuwe, B.E. Housden, C. Chitraju, A.J. Porras, M.M. Graham, X.N. Liu, A.R. Thiam, D.B. Savage, A.K. Agarwal, A. Garg, M.-J. Olarte, Q. Lin, F. Fröhlich, H.K. Hannibal-Bach, S. Upadhyayula, N. Perrimon, T. Kirchhausen, C.S. Ejsing, T.C. Walther, R. V Farese, Seipin is required for converting nascent to mature lipid droplets, Elife. 5 (2016) 1-28. doi:10.7554/elife.16582.

[71] A. Grippa, L. Buxó, G. Mora, C. Funaya, F.-Z.Z. Idrissi, F. Mancuso, R. Gomez, J. Muntanyà, E. Sabidó, P. Carvalho, The seipin complex Fld1/Ldb16 stabilizes ERlipid droplet contact sites, J. Cell Biol. 211 (2015) 829-844. doi:10.1083/jcb.201502070.

[72] C.-W. Wang, Y.-H. Miao, Y.-S. Chang, Control of lipid droplet size in budding yeast requires the collaboration between Fld1 and Ldb16, J. Cell Sci. 127 (2014) 12141228. doi:10.1242/jcs.137737.

[73] V.T. Salo, I. Belevich, S. Li, L. Karhinen, H. Vihinen, C. Vigouroux, J. Magré, C. Thiele, M. Hölttä-Vuori, E. Jokitalo, E. Ikonen, Seipin regulates ER -lipid droplet contacts and cargo delivery , EMBO J. 35 (2016) 2699-2716. doi:10.15252/embj.201695170.

[74] A. Romanauska, A. Köhler, The Inner Nuclear Membrane Is a Metabolically Active Territory that Generates Nuclear Lipid Droplets, Cell. (2018) 1-16. doi:10.1016/j.cell.2018.05.047.

[75] V.T. Salo, S. Li, H. Vihinen, M. Hölttä-Vuori, A. Szkalisity, P. Horvath, I. Belevich, J. Peränen, C. Thiele, P. Somerharju, H. Zhao, A. Santinho, A.R. Thiam, E. Jokitalo, E. Ikonen, Seipin Facilitates Triglyceride Flow to Lipid Droplet and Counteracts Droplet Ripening via Endoplasmic Reticulum Contact, Dev. Cell. (2019). doi:10.1016/j.devcel.2019.05.016.

[76] Y. Cai, J.M. Goodman, M. Pyc, R.T. Mullen, J.M. Dyer, K.D. Chapmana, Arabidopsis SEIPIN proteins modulate triacylglycerol accumulation and influence lipid droplet proliferation, Plant Cell. 27 (2015) 2616-2636. doi:10.1105/tpc.15.00588.

[77] M. Taurino, S. Costantini, S. De Domenico, F. Stefanelli, G. Ruano, M.O. Delgadillo, J.J. Sánchez-Serrano, M. Sanmartín, A. Santino, E. Rojo, SEIPIN proteins mediate lipid droplet biogenesis to promote pollen transmission and reduce seed dormancy, Plant Physiol. 176 (2018) 1531-1546. doi:10.1104/pp.17.01430.

[78] K.D. Chapman, M. Aziz, J.M. Dyer, R.T. Mullen, Mechanisms of lipid droplet biogenesis, Biochem. J. 476 (2019) 1929-1942. doi:10.1042/BCJ20180021.

[79] D. Binns, S. Lee, C.L. Hilton, Q.-X. Jiang, J.M. Goodman, Seipin Is a Discrete Homooligomer, Biochemistry. 49 (2010) 10747-10755. doi:10.1021/bi1013003.

[80] M.F.M. Sim, M.M.U. Talukder, R.J. Dennis, S. O’Rahilly, J.M. Edwardson, J.J. Rochford, Analysis of naturally occurring mutations in the human lipodystrophy protein seipin reveals multiple potential pathogenic mechanisms, Diabetologia. 56 (2013) 2498-2506. doi:10.1007/s00125-013-3029-3.

[81] R. Yan, H. Qian, I. Lukmantara, M. Gao, X. Du, N. Yan, H. Yang, Human SEIPIN Binds 
Anionic Phospholipids, Dev. Cell. $47 \quad$ (2018) 248-256.e4. doi:10.1016/j.devcel.2018.09.010.

[82] X. Sui, H. Arlt, K.P. Brock, Z.W. Lai, F. DiMaio, D.S. Marks, M. Liao, R. V. Farese, T.C. Walther, Cryo-electron microscopy structure of the lipid droplet-formation protein seipin, J. Cell Biol. 217 (2018) 4080-4091. doi:10.1083/jcb.201809067.

[83] I.G. Castro, M. Eisenberg-Bord, E. Persiani, J.J. Rochford, M. Schuldiner, M. Bohnert, Promethin Is a Conserved Seipin Partner Protein, Cells. 8 (2019) 268. doi:10.3390/cells8030268.

[84] M. Eisenberg-Bord, M. Mari, U. Weill, E. Rosenfeld-Gur, O. Moldavski, I.G. Castro, K.G. Soni, N. Harpaz, T.P. Levine, A.H. Futerman, F. Reggiori, V.A. Bankaitis, M. Schuldiner, M. Bohnert, Identification of seipin-linked factors that act as determinants of a lipid droplet subpopulation, J. Cell Biol. 217 (2018) 269-282. doi:10.1083/jcb.201704122.

[85] V. Teixeira, L. Johnsen, F. Martínez-Montañés, A. Grippa, L. Buxó, F.-Z. Idrissi, C.S. Ejsing, P. Carvalho, Regulation of lipid droplets by metabolically controlled Ldo isoforms, J. Cell Biol. 217 (2018) 127-138. doi:10.1083/jcb.201704115.

[86] J. Chung, X. Wu, T.J. Lambert, Z.W. Lai, T.C. Walther, R. V. Farese, LDAF1 and Seipin Form a Lipid Droplet Assembly Complex, Dev. Cell. 51 (2019) 551-563.e7. doi:10.1016/j.devcel.2019.10.006.

[87] S. D'Andrea, Lipid droplet mobilization: The different ways to loosen the purse strings, Biochimie. 120 (2016) 17-27. doi:10.1016/j.biochi.2015.07.010.

[88] E. Boutet, H. El Mourabit, M. Prot, M. Nemani, E. Khallouf, O. Colard, M. Maurice, A.M. Durand-Schneider, Y. Chrétien, S. Grès, C. Wolf, J.S. Saulnier-Blache, J. Capeau, J. Magré, Seipin deficiency alters fatty acid $\Delta 9$ desaturation and lipid droplet formation in Berardinelli-Seip congenital lipodystrophy, Biochimie. 91 (2009) 796-803. doi:10.1016/j.biochi.2009.01.011.

[89] M. Pagac, D.E. Cooper, Y. Qi, I.E. Lukmantara, H.Y. Mak, Z. Wu, Y. Tian, Z. Liu, M. Lei, X. Du, C. Ferguson, D. Kotevski, P. Sadowski, W. Chen, S. Boroda, T.E. Harris, G. Liu, R.G. Parton, X. Huang, R.A. Coleman, H. Yang, SEIPIN Regulates Lipid Droplet Expansion and Adipocyte Development by Modulating the Activity of Glycerol-3phosphate Acyltransferase, Cell Rep. 17 (2016) 1546-1559. doi:10.1016/j.celrep.2016.10.037.

[90] M.M.U. Talukder, M.F.M. Sim, S. O’Rahilly, J.M. Edwardson, J.J. Rochford, Seipin oligomers can interact directly with AGPAT2 and lipin 1, physically scaffolding critical regulators of adipogenesis, Mol. Metab. 4 (2015) 199-209. doi:10.1016/j.molmet.2014.12.013.

[91] M.F.M. Sim, R.J. Dennis, E.M. Aubry, N. Ramanathan, H. Sembongi, V. Saudek, D. Ito, S. O'Rahilly, S. Siniossoglou, J.J. Rochford, The human lipodystrophy protein seipin is an ER membrane adaptor for the adipogenic PA phosphatase lipin 1, Mol. Metab. 2 (2013) 38-46. doi:10.1016/j.molmet.2012.11.002.

[92] W.-C. Su, Y.-H. Lin, M. Pagac, C.-W. Wang, Seipin negatively regulates sphingolipid production at the ER-LD contact site, J. Cell Biol. (2019) jcb.201902072. doi:10.1083/jcb.201902072.

[93] G. van Meer, A.I.P.M. de Kroon, Lipid map of the mammalian cell, J. Cell Sci. 124 (2011) 5-8. doi:10.1242/jcs.071233.

[94] E.R. Rowe, M.L. Mimmack, A.D. Barbosa, A. Haider, I. Isaac, M.M. Ouberai, A.R. Thiam, S. Patel, V. Saudek, S. Siniossoglou, D.B. Savage, Conserved Amphipathic Helices Mediate Lipid Droplet Targeting of Perilipins 1-3, J. Biol. Chem. 291 (2016) 6664-6678. doi:10.1074/jbc.M115.691048. 
[95] A. Čopič, S. Antoine-Bally, M. Giménez-Andrés, C. La Torre Garay, B. Antonny, M.M. Manni, S. Pagnotta, J. Guihot, C.L. Jackson, A giant amphipathic helix from a perilipin that is adapted for coating lipid droplets, Nat. Commun. 9 (2018) 1-16. doi:10.1038/s41467-018-03717-8.

[96] H. Itabe, T. Yamaguchi, S. Nimura, N. Sasabe, Perilipins: a diversity of intracellular lipid droplet proteins, Lipids Health Dis. 16 (2017) 1-11. doi:10.1186/s12944017-0473-y.

[97] J. Bi, Y. Xiang, H. Chen, Z. Liu, S. Grönke, R.P. Kühnlein, X. Huang, Opposite and redundant roles of the two Drosophila: Perilipins in lipid mobilization, J. Cell Sci. 125 (2012) 3568-3577. doi:10.1242/jcs.101329.

[98] Q. Gao, D.D. Binns, L.N. Kinch, N. V Grishin, N. Ortiz, X. Chen, J.M. Goodman, Pet10p is a yeast perilipin that stabilizes lipid droplets and promotes their assembly., J. Cell Biol. 216 (2017) 3199-3217. doi:10.1083/jcb.201610013.

[99] B. Kadereit, P. Kumar, W.-J. Wang, D. Miranda, E.L. Snapp, N. Severina, I. Torregroza, T. Evans, D.L. Silver, Evolutionarily conserved gene family important for fat storage, Proc. Natl. Acad. Sci. 105 (2008) 94-99. doi:10.1073/pnas.0708579105.

[100] D.A. Gross, C. Zhan, D.L. Silver, Direct binding of triglyceride to fat storage-inducing transmembrane proteins 1 and 2 is important for lipid droplet formation, Proc. Natl. Acad. Sci. 108 (2011) 19581-19586. doi:10.1073/pnas.1110817108.

[101] R.D. Moir, D.A. Gross, D.L. Silver, I.M. Willis, SCS3 and YFT2 Link Transcription of Phospholipid Biosynthetic Genes to ER Stress and the UPR, PLoS Genet. 8 (2012) e1002890. doi:10.1371/journal.pgen.1002890.

[102] M. Becuwe, L.M. Bond, N. Mejhert, S. Boland, S.D. Elliott, M. Cicconet, X.N. Liu, M.M. Graham, T.C. Walther, R. V Farese, FIT2 is a lipid phosphate phosphatase crucial for endoplasmic reticulum homeostasis, BioRxiv. (2018) 291765. doi:10.1101/291765.

[103] M.J. Hayes, V. Choudhary, N. Ojha, J.J. Shin, G.-S. Han, G.M. Carman, C.J. Loewen, W.A. Prinz, T.P. Levine, Fat storage-inducing transmembrane (FIT or FITM) proteins are related to lipid phosphatase/phosphotransferase enzymes, Microb. Cell. 5 (2018) 88-103. doi:10.15698/mic2018.02.614.

[104] F.J. Vizeacoumar, J.C. Torres-Guzman, D. Bouard, J.D. Aitchison, R.A. Rachubinski, Pex30p, Pex31p, and Pex32p form a family of peroxisomal integral membrane proteins regulating peroxisome size and number in Saccharomyces cerevisiae., Mol. Biol. Cell. 15 (2004) 665-77. doi:10.1091/mbc.e03-09-0681.

[105] F.J. Vizeacoumar, J.C. Torres-Guzman, Y.Y.C. Tam, J.D. Aitchison, R.A. Rachubinski, YHR150w and YDR479c encode peroxisomal integral membrane proteins involved in the regulation of peroxisome number, size, and distribution in Saccharomyces cerevisiae, J. Cell Biol. 161 (2003) 321-332. doi:10.1083/jcb.200210130.

[106] A.S. Joshi, X. Huang, V. Choudhary, T.P. Levine, J. Hu, W.A. Prinz, A family of membrane-shaping proteins at ER subdomains regulates pre-peroxisomal vesicle biogenesis, J. Cell Biol. 215 (2016) 515-529. doi:10.1083/jcb.201602064.

[107] S. Wang, F.-Z. Idrissi, M. Hermansson, A. Grippa, C.S. Ejsing, P. Carvalho, Seipin and the membrane shaping protein Pex30 cooperate in organelle budding from the endoplasmic reticulum, Nat. Commun. (2018) 1-12. doi:10.1038/s41467-01805278-2.

[108] A.S. Joshi, B. Nebenfuehr, V. Choudhary, P. Satpute-Krishnan, T.P. Levine, A. Golden, W.A. Prinz, Lipid droplet and peroxisome biogenesis occur at the same ER subdomains, Nat. Commun. 9 (2018) 2940. doi:10.1038/s41467-018-05277-3.

[109] S. McConnell, L.C. Stewart, A. Talin, M.P. Yaffe, Temparature-sensitive yeast 
mutants defective in mitochondrial inheritance, J. Cell Biol. 111 (1990) 967-976. doi:10.1083/JCB.111.3.967.

[110] W.M. Henne, L. Zhu, Z. Balogi, C. Stefan, J.A. Pleiss, S.D. Emr, Mdm1/Snx13 is a novel ER-endolysosomal interorganelle tethering protein, J. Cell Biol. 210 (2015) 541551. doi:10.1083/jcb.201503088.

[111] S. Datta, Y. Liu, H. Hariri, J. Bowerman, W.M. Henne, Cerebellar ataxia diseaseassociated Snx14 promotes lipid droplet growth at ER-droplet contacts, J. Cell Biol. 218 (2019) 1335-1351. doi:10.1083/jcb.201808133.

[112] H. Hariri, N. Speer, J. Bowerman, S. Rogers, G. Fu, E. Reetz, S. Datta, J.R. Feathers, R. Ugrankar, D. Nicastro, W.M. Henne, Mdm1 maintains endoplasmic reticulum homeostasis by spatially regulating lipid droplet biogenesis, J. Cell Biol. 218 (2019) 1319-1334. doi:10.1083/jcb.201808119. 


\section{Figure Legends}

\section{Figure 1 - A model for Lipid Droplet biogenesis at the ER}

(A) Neutral lipids (NLs) within the ER bilayer demix forming a lens-like structure (I). Upon growth (II), the LD buds towards the cytosolic face of the ER (III). LDs either remain associated with the ER (III) or completely detach from the ER membrane (IV).

(B) Schematic reprensentation of lipid molecular shapes. Lipids with an inverted cone shape (type I, blue) assemble as positive curved structures (indicated by + ), such as micelles, whereas cone shape lipids (type II, green) form negative curved structures (indicated by -), such as inverted hexagonal phases. Cylindrical-shaped lipids (yellow) adopt bilayer structures. This figure is based on [43].

(C) LD with the ptototypical LD proteins: Class I proteins diffuse from the ER bilayer to the LD and associate with the membrane via a hydrophobic hairpin (green); Class II proteins are recruited from the cytosol and can associate with the LD monolayer, e.g. via an amphipatic helix (orange), protein acylation (burgundy) or protein-protein interactions.

\section{Figure 2 -Major proteins involved in LD biogenesis}

(A) Schematic representation of the synthesis of triacylglycerol (TAG) and steryl-esters (SE). Glycerol-3-phosphate (G3P) is acylated by G3P acyltransferase (GPAT), yielding acyl-G3P or lyso-PA (LPA), which is subsequently acylated by LPA acyltransferase (LPAAT). PA can be hydrolysed to DAG by PA hydrolases (PAH), and the formed DAG is acylated by DGAT enzymes, yielding DAG. For the synthesis of SE, sterols (ST) present in the membrane are $O$-acylated by steryl acyltransferase (SAT). Most acylation steps are substrate dependent on acyl-CoA (indicated by dashed arrows), which can be formed by de novo fatty acid biogenesis (not depicted) or by activation of free fatty acids (FFA) by fatty acid activases (FAA). Metabolic processes (solid black arrows) and enzyme activities (orange) are indicated.

(B) Proteins at the ER domains involved in LD biogenesis; Seipin (Fld1-Ldb16 complex in yeast), FIT proteins (FIT1/2 in mammals; Scs3/Yft2 in yeast), LDO proteins (LDAF1 in mammals, Ldo16/45 in yeast), Pex30 and Perilipins (PLIN). Seipin is depicted as an oligomer.

\section{Figure 3 - Structure of Seipin luminal domain}

(A) Structure of human Seipin luminal domain top (left) and side (right) views. The sideview renders the proposed "docking" of a Seipin oligomer on a budding LD. Stucture was taken from PDB accession number $6 \mathrm{ds} 5$.

(B) Structure of the ER luminal beta-sandwich domain of human (cyan) and fly (blue) Seipin. The structure of $S$. cerevisiae Npc2 (green) bound to an ergosterol molecule (Red) is shown for comparison. Stuctures were taken from PDB accession numbers $6 \mathrm{ds} 5,6 \mathrm{mlu}$, and $6 \mathrm{r} 4 \mathrm{n}$, respectively. 